\title{
Generalized Optoelectronic Model of Series-Connected Multijunction Solar Cells
}

\author{
John F. Geisz, Myles A. Steiner, Iván García, Ryan M. France, William E. McMahon, Carl R. Osterwald, \\ and Daniel J. Friedman
}

\begin{abstract}
The emission of light from each junction in a seriesconnected multijunction solar cell both complicates and elucidates the understanding of its performance under arbitrary conditions. Bringing together many recent advances in this understanding, we present a general 1-D model to describe luminescent coupling that arises from both voltage-driven electroluminescence and voltage-independent photoluminescence in nonideal junctions that include effects such as Sah-Noyce-Shockley (SNS) recombination with $n \neq 2$, Auger recombination, shunt resistance, reverse-bias breakdown, series resistance, and significant dark area losses. The individual junction voltages and currents are experimentally determined from measured optical and electrical inputs and outputs of the device within the context of the model to fit parameters that describe the devices performance under arbitrary input conditions. Techniques to experimentally fit the model are demonstrated for a four-junction inverted metamorphic solar cell, and the predictions of the model are compared with concentrator flash measurements.
\end{abstract}

Index Terms-Electroluminescence (EL), luminescent coupling (LC), multijunction solar cells, photoluminescence (PL).

\section{INTRODUCTION}

$\mathbf{T}$ HE series-connected multijunction solar cell is an optoelectronic device formed from a stack of $\mathrm{p}-\mathrm{n}$ junctions with differing bandgaps interconnected with tunnel junctions, resulting in a simple two-terminal electrical output. Each junction in the stack captures a portion of the incident spectrum with energy greater than its bandgap and passes lower energy photons to subsequent junctions. When a device is properly designed, a nearly equivalent photocurrent is generated in each junction, and photogenerated voltages add to convert a broad solar spectrum into electrical power extremely efficiently. Indeed, conversion efficiencies as high as $46 \%$ have been demonstrated in seriesconnected four-junction devices at high concentration [1]-[4]. This device design has found favor in applications where the high efficiency and simplicity of use are particularly valued, such as space power and high-concentration photovoltaics (HCPV) systems.

However, the internal workings of the series-connected multijunction device are not at all simple, and complete experimental characterization of the device within the framework of a comprehensive optoelectronic model to describe its output under arbitrary input conditions (irradiance, spectrum, temperature, etc.) has been elusive. This type of characterization is particularly important in the case of HCPV systems where the input conditions are continuously changing and yet the true figure of merit is the annual energy production, rather than the efficiency under a single reference spectrum. Such modeling is also useful to understand the fundamental loss mechanisms in multijunction solar cells [5].

The $\mathrm{p}-\mathrm{n}$ junction that is the basic component of these solar cells is the same basic component of light emitting diodes (LED). Considering the reciprocal nature of light emission and collection in a p-n junction [6], it has been said that the best solar cells are also great light emitters [7]. Therefore, as solar cells have improved by reduction of nonradiative recombination, the amount of light emission has also increased. The emitted light both complicates and elucidates the analysis of the equivalent optoelectronic circuit. The effective transfer of current from one junction to another through emitted light is often referred to as radiative or luminescent coupling (LC). The basic phenomenon has been understood for some time now [8], [9], but in recent years, much progress has been made in connecting the basic concepts to the measureable properties of actual multijunction solar cells [10]-[16]. These previous treatments have often assumed a two-diode model with ideality factors of exactly 1 and 2 , which facilitates an analytical treatment. Some of the problems associated with this simple model have been identified through a finite-element analysis of the optical and electrical continuity equations within a typical junction [17]. While an ideality factor of exactly 1 can be justified for recombination in the quasi-neutral region (QNR) under low-injection conditions, there is no physical reason that the Sah-Noyce-Shockley (SNS) recombination mechanism should result in an ideality of exactly 2 [18]-[20]. Device designs that enhance the reabsorption of 
emitted photons within the same junction (photon recycling) through changes in the optical geometry such as the inclusion of back-surface reflectors (BSR) have lead to record-breaking efficiencies in single-junction GaAs solar cells [21], [22] and are under development to improve multijunction devices [23], [24]. The electrical transport properties of the semiconductor have even been shown to be enhanced by photon recycling effects [25], [26]. Additionally, quantitative analysis of the externally emitted light in dark electroluminescence (EL) measurements of multijunction solar cells has been shown to provide essential insight into the internal characteristics of the individual junctions [27]-[29], but previous EL analyses had not accounted for LC that occurs during this "dark" measurement [30]. Finally, voltage-independent photoluminescence (PL) that results from charge separation (without photocurrent collection) in materials of finite mobility [31], [32] has been shown to be an important factor that also affects LC [33].

In this paper, we present a general model that incorporates all the known optical and electrical phenomena that affect the performance of real multijunction solar cells and can be empirically fit to laboratory measurements. While previous work has lumped the LC effect into a single "LC efficiency" which has been defined in multiple ways throughout the literature [13], [14], [16], [17], we separate the various physical phenomena into parameters that can account for multiple types of experiment. The model accounts for LC that arises from both voltage-driven EL and voltage-independent PL and includes SNS recombination with an ideality factor not necessarily equal to 2 , potential Auger recombination, lumped series resistance, junction shunt resistance, and reverse-bias breakdown (RBB). The model can be numerically solved with a straightforward algorithm to describe the entire $J-V$ curve of a multijunction solar cell under arbitrary input conditions. We describe the experimental techniques required to sensitively determine each parameter in the model.

\section{MODEL}

The model described here is a 1-D model that combines important 3-D effects such as distributed series resistance into "effective" parameters. We consider a series-connected multijunction device with a stack of $m$ junctions and label them with subscripts starting at $i=1$ from the illuminated direction. In this approach, we quantify the flow of charge (electrons or holes) and light (photons) between parallel infinite slabs of semiconductor with units of $\left[\mathrm{mA} / \mathrm{cm}^{2}\right]$. The familiar electrical current density units are also applied to photon flux because we take a detailed-balance approach, keeping track of particles that can reversibly be transformed between the two states; a photon can be converted into an electron-hole pair and an electron-hole pair can be converted into a photon. Not all photons are the same, so we bin them by type. For example, we define the flux of photons from an external light source illuminating the front of the multijunction device that are absorbed by the $i$ th junction and collected as photocurrent in that junction

$$
J_{i}^{\text {ext }} \equiv q \int \mathrm{EQE}_{i}(\lambda) \phi^{\text {illum }}(\lambda) d \lambda
$$

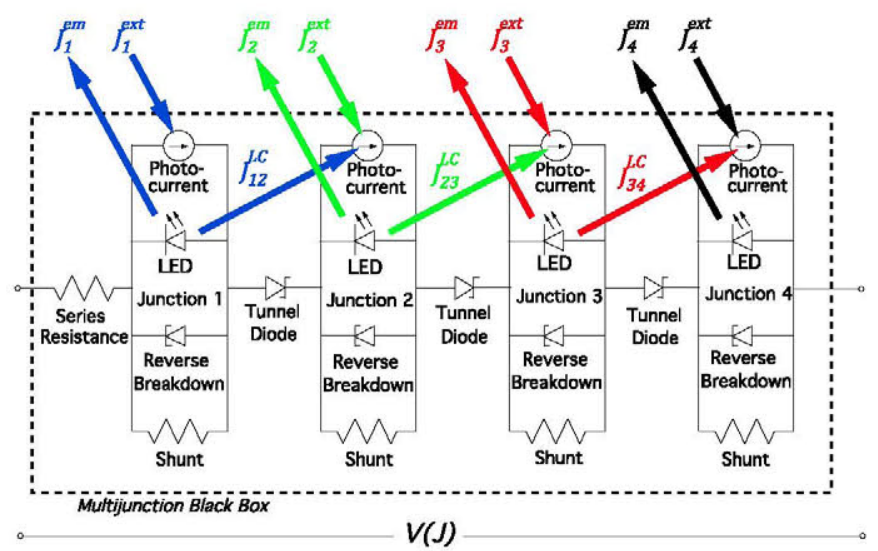

Fig. 1. Schematic of optoelectronic equivalent circuit of a series-connected mutijunction (four junctions here) solar cell showing the measurable input and output channels. It is difficult to electrically probe inside the black box without changing the two-terminal device.

where $q$ is the elemental charge, and $\mathrm{EQE}_{i}$ is the "actual" photovoltaic external quantum efficiency of the $i$ th junction that is a response to external illumination only and has been corrected for artifacts resulting from LC [11], shunting [34], and RBB [35]. $\phi^{\text {illum }}$ is the illuminating spectrum given in units of [photons $/ \mathrm{s} / \mathrm{nm} / \mathrm{cm}^{2}$ ] as a function of wavelength, $\lambda$.

Currently, typical measurements of multijunction solar cells are focused on characterizing the current density versus voltage $(J-V)$ curve of a device under a particular reference spectrum, such as 1-sun AM1.5 global, 1-sun AM0, or AM1.5 direct at some concentration. The premise of these laboratory measurements is that the same $J-V$ curve results for different simulator spectra as long as each junction receives the same external photocurrent, $J_{i}^{\text {ext }}$, as it would under the actual reference spectrum of interest. This is achieved in the laboratory on spectrally adjustable solar simulators using calibrated single-junction reference cells (RCs) and spectral mismatch correction [36]. This treats the multijunction solar cell as a "black box" and provides little information about the performance under differing illumination conditions. Additional insight into what is happening inside the black box is gained by analyzing the only other output that can be measured, the emitted light! A schematic of the optoelectronic model with measureable inputs and output of this black box is shown in Fig. 1 .

\section{A. Emission from Each Junction}

The reciprocal nature of the absorbed and emitted light has been exploited to determine the $J-V$ characteristics of individual junctions within the black box using dark EL measurements [27]-[29]. Initial treatments, however, ignored the considerable LC that can take place during "dark" EL measurements [30]. Furthermore, a voltage-independent mechanism of light emission under illumination (i.e., PL) has recently been recognized to contribute to the emission in superposition (linear combination) with the voltage-driven EL for each junction [31], [32]. The spectrally dependent emission out the front (sun side) of a multijunction solar cell from each junction $i, \Delta \phi_{i}^{e m}$, when it 
is illuminated with a differential photon flux $\Delta \phi_{i}^{\text {illum }}\left(\lambda_{\text {in }}\right)$ at wavelength $\lambda_{\text {in }}$, can be described by

$$
\begin{aligned}
\Delta \phi_{i}^{\text {em }}\left(\lambda_{\text {out }}\right)= & \left.\operatorname{PL}_{i}\left(\lambda_{\text {out }}, \lambda_{\text {in }}\right)\right|_{V=0} \times \Delta \phi_{i}^{\text {illum }}\left(\lambda_{\text {in }}\right) \\
& +\left.\operatorname{EL}_{i}\left(\lambda_{\text {out }}, V\right)\right|_{\text {dark }}
\end{aligned}
$$

where

$$
\begin{aligned}
\left.\mathrm{EL}_{i}\left(\lambda_{\text {out }}, V\right)\right|_{\text {dark }}= & \mathrm{EQE}_{i}\left(\lambda_{\text {out }}\right) \phi_{b b}\left(\lambda_{\text {out }}, T\right) \\
& \times\left[e^{\frac{q V_{i}}{k T}}-1\right] .
\end{aligned}
$$

$\left.\mathrm{PL}_{i}\left(\lambda_{\text {out }}, \lambda_{\text {in }}\right)\right|_{V=0}$ is the voltage-independent PL coupling factor, and $\left.\mathrm{EL}_{i}\left(\lambda_{\text {out }}, V\right)\right|_{\text {dark }}$ is the voltage-dependent, but illumination-independent EL. The reciprocity theorem [6] describes the EL portion of (2), where $\phi_{b b}$ is the black body radiation.

An integration of (2) over all input and output wavelengths allows us to bin the photon flux emitted out the front of the device from each junction $i$ as

$$
J_{i}^{\mathrm{em}}=\gamma_{i} J_{i}^{\mathrm{PC}}+ \begin{cases}J_{i}^{d b}\left[e^{\frac{q V_{i}}{k T}}-1\right], & V_{i} \geq 0 \\ 0, & V_{i}<0\end{cases}
$$

where $k$ is the Boltzmann constant, $T$ is the cell temperature, $V_{i}$ is the voltage across junction $i, \gamma_{i}$ quantifies the voltageindependent PL from a particular junction, and $J_{i}^{\mathrm{PC}}$ is the total photocurrent that is collected in junction $i$. Equation (3) would be more correctly expressed if $J_{i}^{\mathrm{PC}}$ were replaced by the total flux of all photons that are absorbed within the junction (collected as photocurrent or not) but that is difficult to empirically quantify. Rather, we assume that the collection efficiency is good, and that the imperfect carrier collection can be folded into the empirical parameter $\gamma_{i} . J_{i}^{d b}$ is the reverse saturation current in the detailed balance limit of an ideal junction with the absorption characteristics of junction $i$. It is ideal in the sense that only radiative recombination takes place without any parasitic loss of emitted photons which implies a perfect BSR. This critical parameter is the single quantification of the best possible performance of a junction (without using angle restriction [37], [38]) and is the basis for all comparisons with real junctions.

Assuming Lambertian or isotropic emission at the front, $J_{i}^{d b}$ can be calculated simply from the junction $\mathrm{EQE}_{i}$ and the black body emission distribution [6], [39] as

$$
J_{i}^{d b}=\frac{2 \pi q}{h^{3} c^{2}} \int_{0}^{\infty} \mathrm{EQE}_{i}(E) \frac{E^{2}}{\left(e^{\frac{E}{k T}}-1\right)} d E
$$

where $c$ is the speed of light, and $h$ is the Planck constant. While $J_{i}^{d b}$ can, and should, be determined from the "actual" $\mathrm{EQE}$ of the junction (measurements corrected for LC, shunting, and RBB) using (4), it is convenient to relate $J_{i}^{d b}$ to the bandgap, which is more intuitive and easier to use than $J_{i}^{d b}$.

While the bandgap of the junction material is theoretically well defined, uncertainties can arise from traditional methods of extraction from the EQE or PL spectra when, for example, a BSR results in oscillations near the band-edge [39]. Rather, we define the junction bandgap, $\tilde{E}_{i}^{g}$, to be the energy of a stepfunction $\mathrm{EQE}$ that gives the same $J_{i}^{d b}$ as the actual junction. This definition of the junction bandgap is dependent on the optical properties and geometry of the entire device and may not correlate exactly to the bandgap of the junction material (especially for indirect materials). Given a step-function $\mathrm{EQE}$ and neglecting the -1 term in the denominator (which is justified for most bandgaps and temperatures of interest for solar cells), the integration of (4) gives a simple analytical expression [40]:

$$
J_{i}^{d b}\left(\tilde{E}_{i}^{g}, T\right)=\frac{2 \pi q(k T)^{3}}{h^{3} c^{2}}\left[\left(\frac{\tilde{E}_{i}^{g}}{k T}\right)^{2}+2 \frac{\tilde{E}_{i}^{g}}{k T}+2\right] e^{-\tilde{E}_{i}^{g} / k T} .
$$

The inverse function $\tilde{E}_{i}^{g}\left(J_{i}^{d b}, T\right)$ can also be calculated easily by numerically iterating the expression a few times. This relation also provides an important analytical relationship for the temperature dependence for solar cell behavior.

\section{B. Luminescent Coupling Between Junctions}

The total photocurrent $\left(J_{i}^{P C}\right)$ is the result of all light that is absorbed and collected within a junction and includes contributions from external illumination $\left(J_{i}^{\text {ext }}\right)$, as well as internally transferred LC light [13], [14]. Typically, the first junction has a high bandgap, with sequentially lower bandgaps stacked below, so that LC takes place only from the top downward and not upwards, because the earlier junctions are transparent to light emitted by subsequent junctions. In the case of optically thin junctions, significant LC light may be transferred between nonadjacent junctions; therefore, we include it for completeness, but, for the most part, the primary LC will take place between adjacent junctions. The total photocurrent can be expressed as

$$
J_{i}^{P C}=J_{i}^{\text {ext }}+\sum_{k=1}^{i-1} J_{k i}^{\mathrm{LC}} .
$$

Both the LC photocurrent $\left(J_{k i}^{\mathrm{LC}}\right)$ transferred from junction $k$ to junction $i$ and the light emitted from the front of the device $\left(J_{k}^{e m}\right)$ are proportional to the total amount of light emitted from junction $k$. These proportionality constants are determined only by the optical properties and geometry of the structure. By defining average probabilities that a photon emitted from junction $k$ will either 1) escape out the front of the device $\left(P_{k}^{\text {esc }}\right)$, 2) be reabsorbed within the same junction $\left(P_{k}^{\text {abs }}\right)$, or 3 ) be absorbed and collected as photocurrent in another junction $i$ $\left(P_{k i}^{\mathrm{LC}}\right)$, we can relate

$$
J_{k i}^{\mathrm{LC}}=\frac{P_{k i}^{\mathrm{LC}}}{P_{k}^{\mathrm{esc}}} J_{k}^{e m}=\beta_{k i} J_{k}^{e m} .
$$

While $P_{k}^{\text {esc }}, P_{k}^{\text {abs }}$, and $P_{k i}^{\mathrm{LC}}$ may be estimated by optical modeling of the layered stack as described in [21] and [41], they are difficult to measure directly. The geometrical optical coupling parameter $\left(\beta_{k i}\right)$, on the other hand, is an empirical parameter that can be determined experimentally. $\beta_{k i}$ should be a constant as long as the optical geometry remains constant. If the location of emission within the junction or the optical material parameters change (e.g., with T), $\beta_{k i}$ may change because 
that changes the optical geometry. For example, the spatial distribution of emission within the junction may be different between the PL $\left.\right|_{V=0}$ and EL $\left.\right|_{\text {dark }}$ mechanisms of emission [33], but to first approximation, we will assume that $\beta_{k i}$ is constant at a given temperature. For adjacent optically thick junctions, $\beta_{k, k+1} \approx n^{2}$, where $n$ is the index of refraction of the semiconductor layers, but can be as high as $\beta_{k, k+1} \approx 4 n^{2}-1$ for optically thin junctions [21]. Note that imperfect collection efficiency within junction $i$ or parasitic absorption between the junctions will reduce $\beta_{k i}$ from these upper limits.

While the analysis presented here does not rely on the independent values of $P_{i}^{\text {esc }}$ and $P_{i}^{\text {abs }}$ for each junction, it is interesting to note that they can be used to relate the external radiative efficiency $\left(\eta_{i}^{\text {ext }}\right)$ of each junction $i$ to the internal radiative efficiency $\left(\eta_{i}^{\text {int }}\right)$ [7], [21] as

$$
\eta_{i}^{\text {ext }}=\frac{\eta_{i}^{\text {int }} P_{i}^{\mathrm{esc}}}{1-\eta_{i}^{\text {int }} P_{i}^{\text {abs }}}
$$

This internal radiative efficiency is an absolute metric of the junction quality at a given current density. The relation is also useful to provide upper limits of the external radiative efficiency for a given optical geometry and can define an internal radiative limit $[42]\left(\eta_{i}^{\text {int }}=1\right)$ that is more realistic than the overly optimistic detailed balance limit without any photon loss $\left(\eta_{i}^{\text {ext }}=1\right)$. The external radiative limit implies that there can be no $\operatorname{LC}\left(\beta_{i k}=0\right)$ or parasitic photon absorption of any kind, since all emitted light must escape out the front of the device. The internal radiative limit in the absence of a perfect reflector, on the other hand, requires some photon loss such as LC and places a lower limit on $J_{i}^{01}$ :

$$
\frac{J_{i}^{01}}{J_{i}^{d b}} \geq \frac{1-P_{i}^{\mathrm{abs}}}{P_{i}^{\mathrm{esc}}} \geq 1+\sum_{k>i} \beta_{i k} .
$$

This relationship can be used to derive the classical solutions for the internal radiative limit of individual junctions in specific geometrical cases. Three cases are outlined in [37]: Structure I is the case of an optically thick junction on an absorbing substrate which is equivalent to a subsequent absorbing junction (described by Henry [43]) and results in $J_{i}^{01}=\left(1+n^{2}\right) J_{i}^{d b}$ using the limiting values of $P_{i}^{\text {esc }}$ and $P_{i}^{\text {abs }}$ given in [21]. Structure II is the case of a perfect BSR that gives $J_{i}^{01}=J_{i}^{d b}$. Structure III is the case of an ideal bifacial cell (treated by Shockley-Queisser [44]) that gives $J_{i}^{01}=2 J_{i}^{d b}$ since photon escape is equally likely from the front and back of the cell.

\section{Electrical Circuit}

In a series-connected multijunction solar cell, the voltage across the entire device, $V$, for a given current density, $J$, is just the sum of the voltages of each series-connected component including the equivalent series resistance [12]:

$$
V(J)=J \cdot R^{\mathrm{ser}}+\sum_{i=1}^{m} V_{i}(J)
$$

where $V_{i}(J)$ is the voltage across the $i$ th junction, and $R^{\text {ser }}$ is the lumped series resistance, which accounts for all contact resistances, sheet resistances, effective resistances across

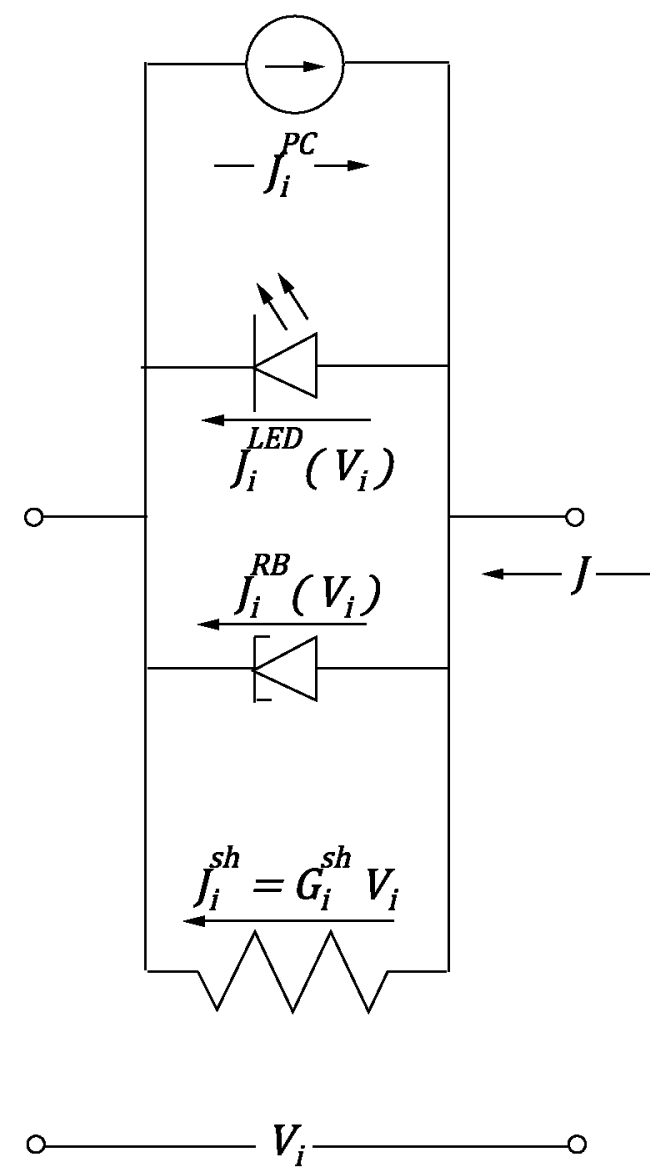

Fig. 2. Schematic of electrical equivalent circuit of each junction $i$ within a multijunction solar cell.

all tunnel junctions (well below their peak tunneling current) and other heterobarriers, etc. We do not treat the behavior at currents greater than the peak tunneling currents or the effect of distributed series resistance here.

The equivalent model of each junction includes several elements occurring in parallel, such as a current source from the photocurrent, the LED, a reverse breakdown diode, and a shunt resistance. The sum of all of these parallel currents within each junction must equal the total current flowing through the entire series-connected device, as shown in Fig. 2:

$$
-J_{i}^{\mathrm{PC}}+J_{i}^{\mathrm{LED}}+J_{i}^{\mathrm{RB}}+G_{i}^{\mathrm{sh}} V_{i}=J
$$

where $J_{i}^{\mathrm{LED}}$ is the recombination current through the diode, $J_{i}^{\mathrm{RB}}$ is the current through an RBB diode, and $G_{i}^{\text {sh }}$ is the conductance of a shunt resistor across the ith junction. The total photocurrent, $J_{i}^{\mathrm{PC}}$, which flows counter to the other currents, includes a negative sign here so that its value will be positive.

Equation (11) [inserting (6)] can be divided into two current terms: a source or injection current and a sink or recombination current and reexpressed as

$$
J_{i}^{\mathrm{inj}}=J_{i}^{\mathrm{rec}}
$$


where

$$
J_{i}^{\mathrm{inj}} \equiv J+J_{i}^{\mathrm{ext}}+\sum_{k=1}^{i-1} J_{k i}^{\mathrm{LC}}
$$

and

$$
J_{i}^{\mathrm{rec}} \equiv J_{i}^{\mathrm{LED}}\left(V_{i}\right)+J_{i}^{\mathrm{RB}}\left(V_{i}\right)+G_{i}^{\mathrm{sh}} V_{i} .
$$

The solution for $V_{i}(J)$ relies on an appropriate model for each component of these parallel currents. A fairly comprehensive model for the LED current in the junction is given by a three-diode model [13] that incorporates a term for 1) radiative and nonradiative recombination in the QNR, 2) Shockley-ReadHall nonradiative recombination within the junction (aka SNS recombination), and 3 ) Auger recombination. The reverse saturation current densities for each of these recombination mechanisms in junction $i$ are given by $J_{i}^{01}, J_{i}^{0 n}$, and $J_{i}^{o o}$, respectively. The total LED current is then

$$
\begin{aligned}
J_{i}^{\mathrm{LED}}\left(V_{i}\right)= & J_{i}^{01}\left[e^{\frac{q V_{i}}{k T}}-1\right]+J_{i}^{0 n}\left[e^{\frac{q V_{i}}{n^{\frac{1}{2} \mathrm{~N}} k T}}-1\right] \\
& +J_{i}^{00}\left[e^{\frac{3 q V_{i}}{2 k T}}-1\right]
\end{aligned}
$$

where the SNS ideality factor $n_{i}^{\text {SNS }}$ is not necessarily equal to 2 [45]. This model can be improved with any other terms needed to capture the physics of important recombination mechanisms for a given device as long as the recombination current is given explicitly as a function of junction voltage.

In multijunction solar cells, the effects of RBB can be observed in the power-producing quadrant whenever the onset of breakdown of the limiting junction is greater than the sum of the other junction voltages [35], [46]. The effects of RBB are observed increasingly for junctions with low bandgap and high doping, such as germanium and 0.7-eV InGaAs junctions. The mechanism of breakdown can be avalanche, tunneling, or both. Here, we model RBB empirically as a generic diode operating in the opposite direction with a large ideality factor $n_{i}^{\mathrm{RB}}$ :

$$
J_{i}^{\mathrm{RB}}\left(V_{i}\right)= \begin{cases}-J_{i}^{\mathrm{ORB}}\left[e^{\frac{-q V_{i}}{n_{i}^{\mathrm{RB}} k_{i} T}}-1\right], & V_{i} \leq 0 \\ 0, & V_{i}>0 .\end{cases}
$$

Alternative models of the RBB have been proposed that invoke an avalanche multiplication factor [47]. While these other models may have slightly better physical justification, they require fitting of more parameters without significant improvement in the fit. Further investigation into the applicability of these models to particular materials and junctions may be important, for example, to predict the temperature-dependent behavior of RBB.

Inserting (13) and (14) into (12) results in a single nonlinear equation that can be numerically solved for $V_{i}(J)$ by searching over a rather small range of voltages for external currents, $J$, of interest. Because the LC current is almost always one-way by nature, each junction voltage may be solved successively starting with the top junction (where the photocurrent is not affected by LC), using LC photocurrents that depend only on the light emission of previously calculated junctions using (3), (6), and (7). In the case of $n_{i}^{\text {SNS }}=2$, negligible Auger recombination, reverse breakdown, and shunt conductance, the nonlinear equation for $V_{i}(J)$ has been solved analytically [12].

\section{Departures from One-Dimensional Model}

Concentrator multijunction solar cells are generally designed for systems that focus light onto only a portion of the device, leaving relatively large areas of the device unilluminated. The unilluminated portions of the device contain large busbars to handle the high currents generated in the relatively small device. Consequently, even in laboratory measurements under a uniform collimated solar simulator, a relatively large fraction of the device area may be shadowed from the external light. Measurement of concentrator devices under the G173 direct specification allows the busbar area to be subtracted from the total device area ( $A^{\text {total }}$ ) scaling the current density by only the illuminated area $\left(A^{\text {illum }}\right)$. If this area definition is used, the dark area of the device reduces the voltage by up to $\Delta V_{\mathrm{oc}}=\frac{2 m k T}{q} \ln \left(A^{\text {illum }} / A^{\text {total }}\right)$ from the predictions of the above model, where $m$ is the number of junctions. Improved modeling of the $V_{\text {oc }}$ is achieved if we perform the calculation based on the total area and assume that the external photocurrent that is unshadowed spreads laterally to provide a uniform distribution. Therefore, we scale both $J_{i}^{\text {ext }}$ and $J$ by $\left(A^{\text {illum }} / A^{\text {total }}\right)$ to perform the calculation and translate the results to fit data based on the standard measurement area.

The lumped series resistance of this model incorporates many sources of resistance including heterojunction barriers, tunnel junctions, and laterally distributed resistances defined by the grid geometry. The series resistance becomes important at high current densities in both dark $J-V$ and concentrated flash measurements, but the direction and path of current flow are quite different between these measurements. Therefore, it is not surprising to find that very different $R^{\text {ser }}$ values are required to fit the dark $J-V$ and flash measurements. When the series resistance first begins to become important in these experiments, a "lumped" series resistance may be sufficient to qualitatively describe the behavior, but at very high currents, the voltage distribution and current flows can vary significantly over lateral dimensions, requiring a full 3-D implementation of these principles, which is beyond the scope of this paper.

\section{Experimental Determination of Cell Parameters}

No single experiment is sufficient to completely characterize this entire complex optoelectronic model of a multijunction solar cell. Dark EL measurements are very sensitive to the voltagerelated characteristics of the subcells, and measurements of $J_{\mathrm{sc}}$ while varying the external illumination are sensitive to the subcell currents and LC. Previous literature treated these two experiments separately, but the full model parameters are interrelated. By fitting the results of the following set of experiments to the full model simultaneously, a given multijunction solar cell can be fully characterized. The true test of this model for a particular device is that these and other measurement results can be completely described with a single set of parameters. In this section, we describe and fit a suite of measurements that is 
sensitive to all the model parameters using an example of a fourjunction inverted metamorphic (4J IMM) concentrator solar cell [1]. This 4J IMM device incorporates high radiative efficiency rear-heterojunction designs in the top two junctions [42], [48].

The following measurements, each of which has been described previously in the literature as an independent measurement, are performed together and fit to the model simultaneously:

A) dark EL [28], [29], [48]

$J_{i}^{\text {em }}\left(J ; J_{i}^{\text {ext }}=0\right)$;

B) limiting photocurrent under varied illumination [11], [13] $J\left(J_{i}^{\text {ext }} ; V \approx 0\right)$;

C) emitted light under varied illumination $J_{i}^{\text {em }}\left(J_{i}^{\text {ext }} ; V \approx 0\right)$.

The measurements are performed within a single solar simulator apparatus that consists of multiple independently controlled, collimated, high-intensity LED light sources [11], a calibrated spectroradiometer to quantitatively measure the emitted and illuminating light spectrum [48], isotype RCs that are calibrated to the standard reference spectrum (e.g. AM1.5 global, AM1.5 direct, or AM0), and a high precision source meter. Since concentrator solar cells and the corresponding RCs are typically relatively small $\left(0.1 \mathrm{~cm}^{2}\right.$ in the example), we only need homogenous illumination over a relatively small area, but all light sources must be pointed to a single spot in space $(x, y, z$ dimension) which can be reproducibly accessed by the device under test (DUT), RCs, and spectroradiometer. An additional measurement (see Section III-D) is performed separately only to test the predictions of the model parameters that are fit using the first three measurements.

The model is strongly dependent on the detailed balance reverse saturation current density, $J_{i}^{d b}$, through (3). This parameter is not fit to the experiments described above, but is directly computed from the $\mathrm{EQE}_{i}$ using (4). In addition, the $\mathrm{EQE}$ must be known before the illumination can be quantified for experiments B and C. EQE measurements of each junction can be performed using relatively standard equipment with voltage- and light-biasing capabilities. Continuously operated LEDs (dc) with wavelengths chosen to match each junction are used to overilluminate all but the junction of interest, while the monochromatic probing light (ac) is chopped at $\sim 300 \mathrm{~Hz}$. Artifacts arising from shunts [34] and RBB [35] are reduced through voltage-biasing based on $J-V$ curves measured under the $\mathrm{EQE}$ testing conditions. The effects of $\mathrm{LC}$ on the $\mathrm{EQE}$ are very real and are affected by the measurement conditions. The "actual" $\mathrm{EQE}$, which is the response only to the external illumination and not luminescence from another junction, is determined numerically using the equations in the appendix of [11] by assuming that the "actual" EQE approaches zero asymptotically at short wavelengths for junctions with optically thick filtering junctions at those short wavelengths. Applying this criterion over a range of wavelengths rather than at a single wavelength reduces the effects of measurement noise. Similar numerical correction has also been shown to apply for any remaining artifacts of shunting [49]. The spectral correction to the combination of LC and shunting effects could also be determined using a pulsed light or voltage analysis at a few distinct wavelengths [50]-[52].

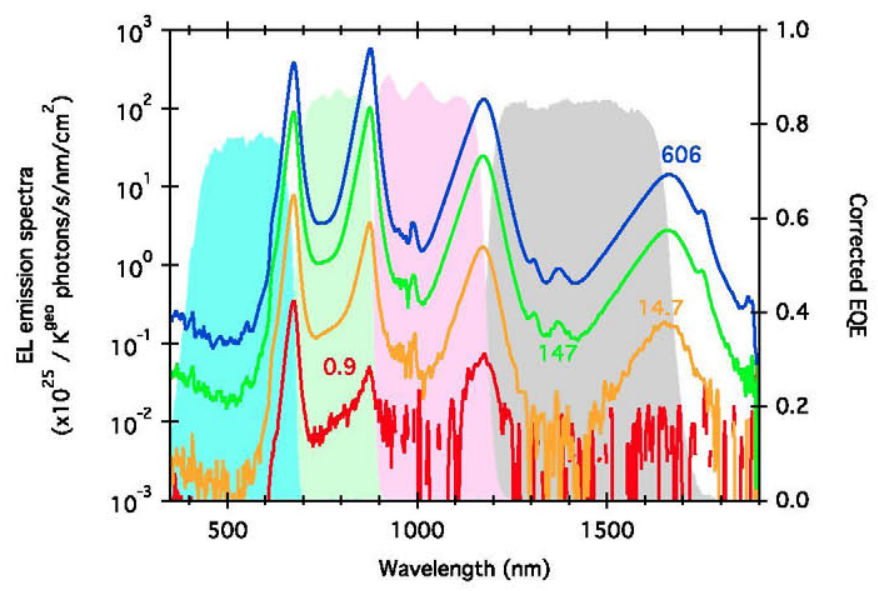

Fig. 3. External quantum efficiency (filled curves) and dark EL emission (lines) for various external current injections of the 4J IMM. The injected current density $\left(\mathrm{mA} / \mathrm{cm}^{2}\right)$ of the colored lines is labeled with the same color.

The fitting procedure used here involves iteratively adjusting the parameters while interactively comparing the model with the measured data from all three experiments. This suite of measurements is chosen specifically as a minimum set of experiments that will uniquely determine all the parameters through fitting. Because the experiments have different sensitivities to different parameters, the interdependent parameters can be determined in just a few iterations if good initial guesses for the parameters are chosen. The model parameters that are most sensitive to each measurement are described in detail within each following section.

\section{A. Dark Electroluminescence Measurements}

By electrically forward biasing a solar cell in the dark with current, $J$, and measuring the emitted light spectrum, $\phi^{\mathrm{em}}(\lambda)$, in absolute units of photons $/ \mathrm{s} / \mathrm{nm} / \mathrm{cm}^{2}$, we can experimentally determine

$$
J_{i}^{\mathrm{em}}=\frac{q}{K^{\mathrm{geo}}} \int_{\lambda_{i}^{\min }}^{\lambda_{i}^{\max }} \phi_{\mathrm{meas}}^{\mathrm{em}}(\lambda) d \lambda
$$

for each junction from EL because the emission is typically a relatively narrow spectral band around the direct bandgap of each junction, thereby allowing easy identification of the junction of origin from spectrally resolved measurements. The absolute calibration of the emitted spectrum can be made assuming an infinite plane of Lambertian emission [48], but the finite area of the solar cell and the geometry of light collection requires an additional wavelength-independent constant, $K^{\mathrm{geo}}$, for final absolute calibration. Fig. 3 shows the measured EL emission from a 4J IMM along with the corrected EQE. From these spectra, individual subcell voltages $V_{i}(J)$ can be determined over a wide range of $J$ by rearranging (3):

$$
V_{i}=\frac{k T}{q} \ln \left\{\left[J_{i}^{\mathrm{em}}-\gamma_{i} \sum_{k=1}^{i-1} \beta_{k i} J_{k}^{\mathrm{em}}\right] / J_{i}^{d b}+1\right\} .
$$

The sum of the calculated junction voltages by (10) can then be compared with the electrically measured dark current $V(J)$ 
to determine $K^{\text {geo }}$. This comparison must be done below the current density where series resistance becomes dominant, but at high enough currents to measure the luminescent spectra with good signal-to-noise ratio. It is important to note that while EL is nominally performed in the dark ( $\left.J_{i}^{\text {ext }}=0\right)$, the light emitted from one junction can strongly illuminate other junctions $\left(J_{k i}^{\mathrm{LC}} \neq 0\right)$, complicating the analysis. This effect can result in a much higher injection current in the second and subsequent junctions than the externally applied dark current, but it can be calculated by combining (7) and (12b):

$$
J_{i}^{\mathrm{inj}}=J+\sum_{k=1}^{i-1} \beta_{k i} J_{k}^{\mathrm{em}}
$$

using the EL measured emission of other junctions and the empirical optical coupling parameter $\beta_{k i}$ which can be determined experimentally by simultaneous fitting of the subsequent LC measurements. With the strong forward bias applied in EL and cascading LC, the actually injection current of the $i$ th junction could be almost as large as $i \times J$ for strong LC [30]. The effects of coupled light emission during dark EL measurements on the voltage determined at that measurement condition using (16) are much smaller than the effects on the current using (17), since they only occur due to the second-order effect of PL at short circuit (i.e., $\gamma_{i}>0$ ). The experimental values of $V_{i}, J_{i}^{\text {inj }}$, and the external radiative efficiency $\eta_{i}^{\text {ext }} \equiv \frac{J_{i}^{\text {em }}}{J_{i}^{\text {inj }}}$ are calculated from the measured set of $J_{i}^{\mathrm{em}}(J)$ assuming model parameters of $\beta_{k i}$ and $\gamma_{i}$ that will be fit more sensitively by the measurements described in Sections III-B and -C, respectively. While the EL measurements provide data that are unaffected by series resistance, the effect of junction shunt resistance is well captured. Thus, from each $V_{i}\left(J_{i}^{\mathrm{inj}}\right)$ or $\eta_{i}^{\mathrm{ext}}\left(J_{i}^{\mathrm{inj}}\right)$ curve, we can sensitively fit the subcell diode parameters from (13), such as $J_{i}^{01}, J_{i}^{0 n}, n_{i}^{\mathrm{SNS}}$, as well as the shunt conductance. $E_{i}^{g}\left(J_{i}^{d b}, T\right)$ defined by (5) and ratios relative to $J_{i}^{d b}$ are easier to adjust while fitting than the values that vary exponentially with $E_{i}^{g} / k T$ such as $J_{i}^{01}, J_{i}^{0 n}$, etc. $\eta_{i}^{\text {ext }}\left(J_{i}^{\text {inj }}\right)$ is particularly sensitive [27], [48] to $J_{i}^{01} / J_{i}^{d b}$ (at high currents), to $J_{i}^{0 n} /\left(J_{i}^{d b}\right)^{1 / n}$ (at low currents), and to $n_{i}^{\text {SNS }}$ (at low currents). All of these convenient ratios are used in Table I to summarize the fit. Note that all four subcells are fit with $n_{i}^{\mathrm{SNS}}<2$. A good fit to the electrical $J-V$ curve at very low currents is an additional check on $\sum n_{i}^{\mathrm{SNS}}$. Fig. 4 shows the EL data of the 4J IMM represented in terms of (a) $V_{i}\left(J_{i}^{\text {inj }}\right)$, (b) $\eta_{i}^{\text {ext }}\left(J_{i}^{\text {inj }}\right)$, and (c) $\eta_{i}^{\text {int }}\left(J_{i}^{\text {inj }}\right)$. The large open markers are the EL data calculated using (15)-(17), along with the model fit (lines) using the final fit parameters given in Table I. The internal radiative efficiency was calculated using the values of $P_{k}^{\text {esc }}$ and $P_{k}^{\text {abs }}$ taken from [42]. The smaller filled markers in Fig. 4(b) and (c) show the significant errors that result when $\mathrm{LC}$ during $\mathrm{EL}$ is neglected (i.e., $\beta_{k i}=0$ ). Without considering $\mathrm{LC}$, the internal radiative efficiency exceeds one for the excellent GaAs junction, giving an unphysical result. In this 4J IMM, we could not achieve high enough current densities to observe any effect of Auger recombination.

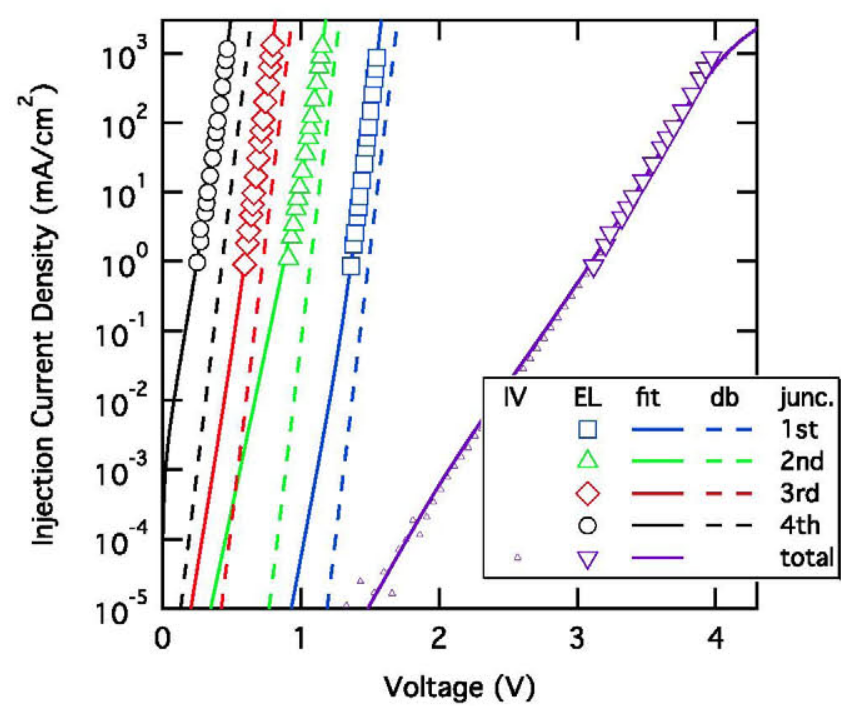

(a)

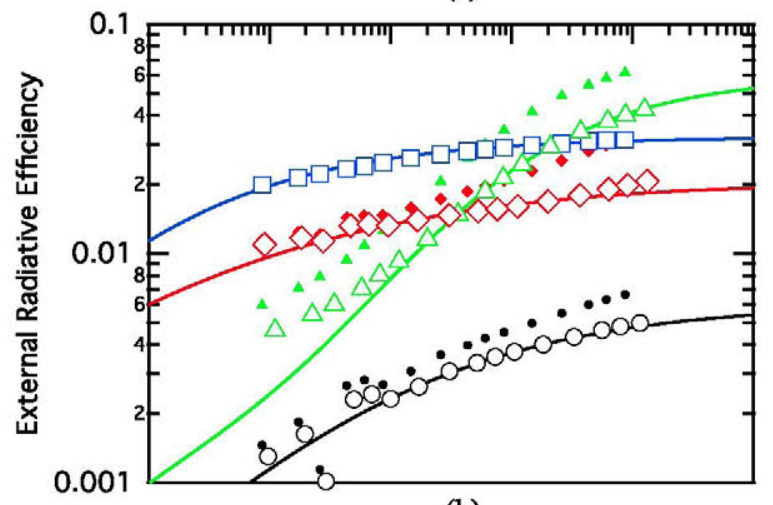

(b)

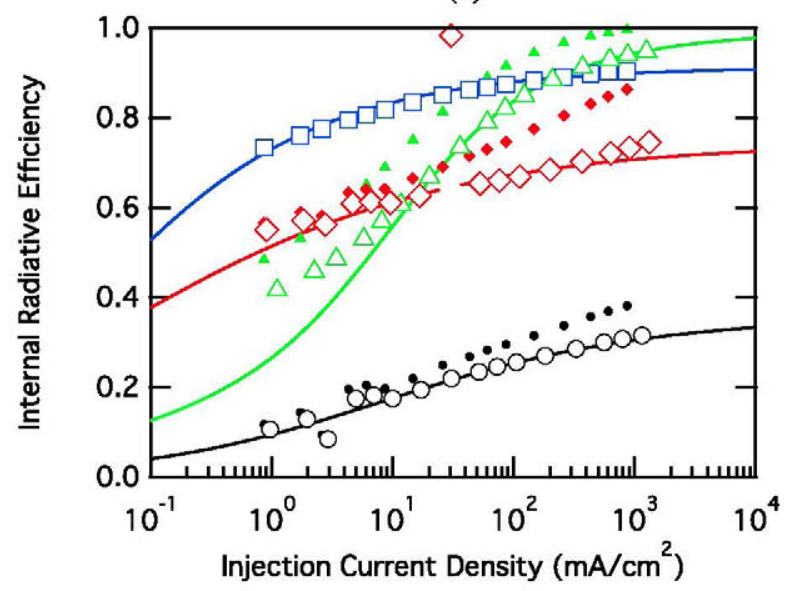

(c)

Fig. 4. EL data presented as (a) $V_{i}\left(J_{i}^{\mathrm{inj}}\right)$ and total $V(J)$ dark $J-V$ curves, (b) external radiative efficiency, and (c) internal radiative efficiency. The solid lines show the model predictions using the parameters in Table I, the large open markers are calculated from the EL data using only $\gamma_{\mathrm{i}}$ and $\beta_{i, i+1}$ from the Table.

\section{B. Measurement of Limiting Photocurrent While Varying External Illumination on each Junction Independently}

The measurement and fitting to the simple two-diode model of LC has been covered extensively in the literature [11]-[13] using 
TABLE I

Fitting Parameters Used to Calculate All Model Predictions

\begin{tabular}{lcccc}
\hline \hline Junction $i$ & 1 & 2 & 3 & 4 \\
\hline$\tilde{E}_{i}^{g}(\mathrm{eV})$ & 1.830 & 1.404 & 1.049 & 0.743 \\
$J_{i}^{1 X}\left(\mathrm{~mA} / \mathrm{cm}^{2}\right)$ & 11.96 & 11.49 & 11.35 & 12.28 \\
$J_{i}^{Q \mathrm{E}}\left(\mathrm{mA} / \mathrm{cm}^{2}\right)$ & 12.76 & 12.49 & 11.85 & 11.38 \\
$J_{i}^{01} / J_{i}^{d b}$ & 31 & 17 & 51 & 173 \\
$n_{i}^{\mathrm{SN} \mathrm{N} \mathrm{S}}$ & 1.6 & 1.8 & 1.4 & 1.5 \\
$J_{i}^{0 n} /\left(J_{i}^{d b}\right)^{1 / n_{i}^{S \mathrm{SS}}}\left({\left.\mathrm{mA} / \mathrm{cm}^{2}\right)^{(1-\mathrm{n}) / \mathrm{n}}}\right.$ & 4.5 & 42 & 14 & 79 \\
$J_{i}^{0 a} /\left(J_{i}^{d b}\right)^{3 / 2}\left(\mathrm{~mA} / \mathrm{cm}^{2}\right)^{-1 / 2}$ & 0 & 0 & 0 & 0 \\
$n_{i}^{\mathrm{RB}}$ & 0 & 0 & 0 & 46 \\
$J_{i}^{0 \mathrm{RB}} /\left(J_{i}^{d b}\right)^{1 / n}{ }_{i}^{\mathrm{RB}}\left(\mathrm{mA} / \mathrm{cm}^{2}\right)^{(1-\mathrm{n}) / \mathrm{n}}$ & 0 & 0 & 0 & 0.3 \\
$\gamma_{i}$ & 0.004 & 0.006 & 0.0022 & 0.001 \\
$G_{i}^{s h}\left(\Omega^{-1} \mathrm{~cm}^{-2}\right)$ & 0 & 0 & 0 & 0 \\
$\beta_{i, i+1}$ & 14.3 & 8.6 & 10.5 & - \\
\hline \hline
\end{tabular}

The model in Section II is fit to the experiments presented in Sections III-A-III-C for 4J IMM solar cell (MM927n10) as shown in Figs. 4-7. T $=25^{\circ} \mathrm{C}, K^{g 00}=$ $0.86, A^{\text {illum }} / A^{\text {total }}=0.869, R^{\text {er }}($ dark $J-V)=0.10 \Omega \cdot \mathrm{cm}^{2}, R^{\text {ser }}($ Flash $)=$ $0.015 \Omega \cdot \mathrm{cm}^{2} \cdot J_{i}^{d b}\left(\tilde{E}_{i}^{g}, T\right)$ is given by (5).

the parameters $\eta_{k i}^{\mathrm{LC}} \equiv \beta_{k i} \frac{J_{k}^{d b}}{J_{k}^{01}}$ and $\varphi_{i} \equiv \frac{J_{i}^{02}}{2 \sqrt{J_{i}^{01}}}$ (defined relative to the parameters used here). These parameters are sufficient to describe the simpler model with $n_{i}^{\text {SNS }}=2$ when only this measurement is being fit, but the uncertainties were high because multiple combinations of $\eta_{k i}^{\mathrm{LC}}$ and $\varphi_{i}$ could be found to fit this single measurement fairly well. By simultaneously fitting the EL data, the uncertainty is dramatically reduced, but to do this, it is necessary to separate the emission properties of the junction from the optical geometric coupling factor-motivating the change in nomenclature used here. The experimental technique involves measuring $J-V$ curves (or just the limiting photocurrent that would be $J_{\mathrm{sc}}$ in the absence of RBB or shunts) while systematically varying the illuminating spectrum to independently and quantitatively vary $J_{i}^{\text {ext }}$, and fitting the data to a model that includes LC. The definition of $J_{i}^{\text {ext }}$ in (1) relies on absolutely accurate EQE and illumination spectra, but the uncertainty can be reduced by relating it to the suns of illumination on each junction, $X_{i}$, relative to the 1-sun external photocurrent of that junction within the multijunction device for a given reference spectrum, $J_{i}^{1 X}$, which we leave as a fitting parameter

$$
J_{i}^{\text {ext }}=J_{i}^{1 X} \cdot X_{i} .
$$

The illumination relative to a particular reference spectrum is quantified by measuring a calibrated RC and the spectrum at each measurement illumination condition and calculating $X_{i}$ making use of a spectral mismatch correction factor [11], [36]

$$
\begin{aligned}
X_{i}= & \frac{I_{\mathrm{RC}}^{\text {illum }}}{I_{\mathrm{RC}}^{\text {refspec }}} \\
& \times \frac{\int \mathrm{EQE}_{i}^{\mathrm{DUT}}(\lambda) \phi^{\text {illum }}(\lambda) d \lambda \cdot \int \operatorname{EQE}^{\mathrm{RC}}(\lambda) \phi^{\text {refspec }}(\lambda) d \lambda}{\int \mathrm{EQE}_{i}^{\mathrm{DUT}}(\lambda) \phi^{\text {refspec }}(\lambda) d \lambda \cdot \int \operatorname{EQE}^{\mathrm{RC}}(\lambda) \phi^{\text {illum }}(\lambda) d \lambda}
\end{aligned}
$$

where $I_{\mathrm{RC}}^{\mathrm{illum}}$ is the measured short-circuit current of the RC, and $I_{\mathrm{RC}}^{\mathrm{refspec}}$ is the calibrated value of that RC under the reference spectrum $\phi^{\text {refspec }}(\lambda)$. If the EQE of the RC is perfectly matched to the DUT, the integrals cancel out, but even if they

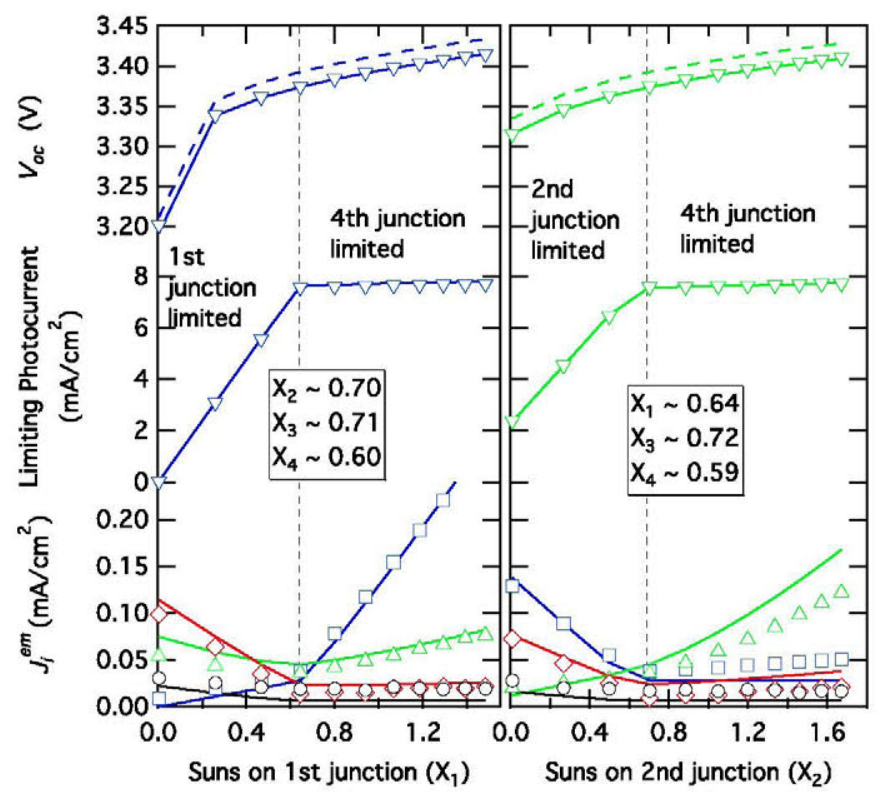

(a)

(b)

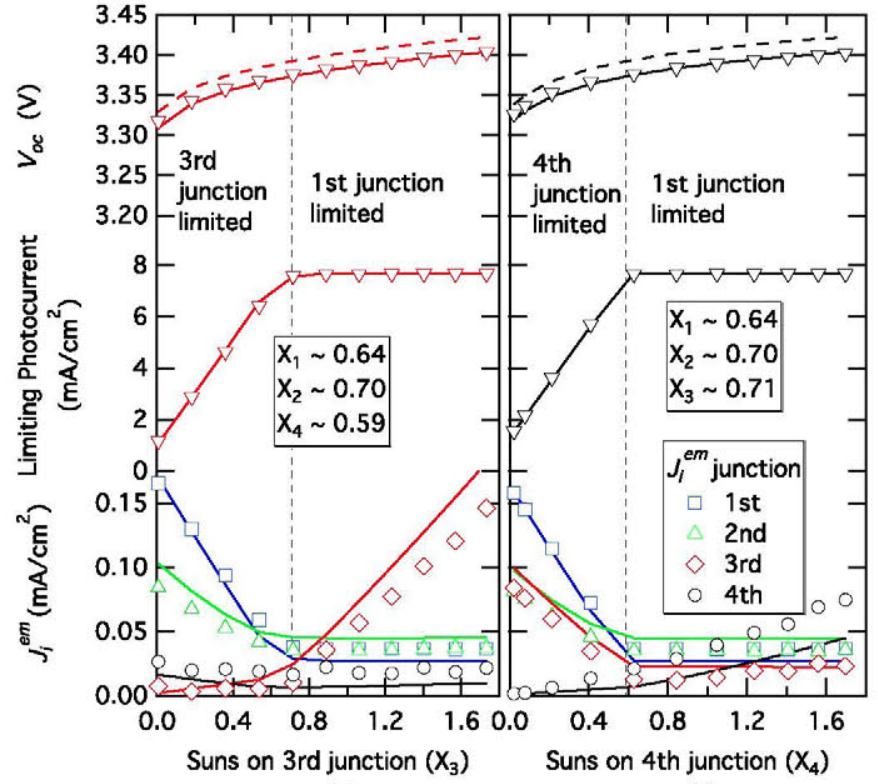

(c)

(d)

Fig. 5. "Light" varied illumination measurements. Total $V_{o c}$, the limiting photocurrent $=J(2.7 \mathrm{~V})$, and light emitted from each junction, $J_{i}^{e m}$, is shown as the external illumination is varied around 0.7 suns (relative to AM1.5 direct). The inset legends show the approximate illumination on the junctions that are not intentionally varied. Data (markers) fit using parameters in Table I (solid lines). The $V_{o c}$ is overestimated when the dark area correction is not performed (colored dashed lines). The external photocurrent supplied to the first junction is varied in (a), the second junction is varied in (b), the third junction is varied in (c), and the fourth junction is varied in (d). The black vertical dashed line indicates where the limiting junction changes.

are not matched, the uncertainty is reduced since the absolute magnitude of both the EQE and illumination spectrum cancel out so that only the shapes of the EQE and spectra are important.

In order to sensitively fit the parameters $J_{i}^{1 X}, \beta_{k i}$ and the parameters that describe the nonradiative recombination in (11), the spectrum must be varied such that each junction is forced to be the limiting current junction. Fig. 5 shows the limiting photocurrent (at $V=2.7 \mathrm{~V}$ ) and $V_{\mathrm{oc}}$ as the illumination is varied 


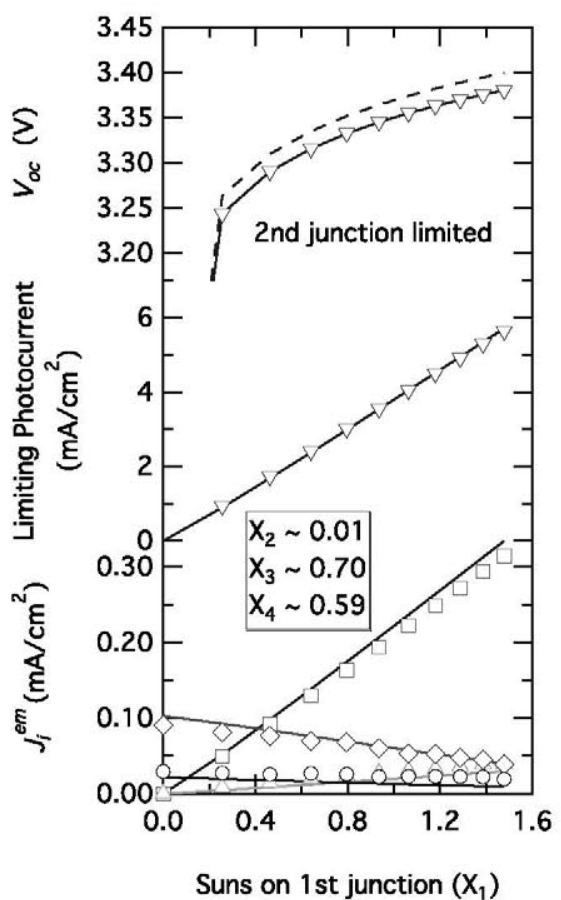

(a)

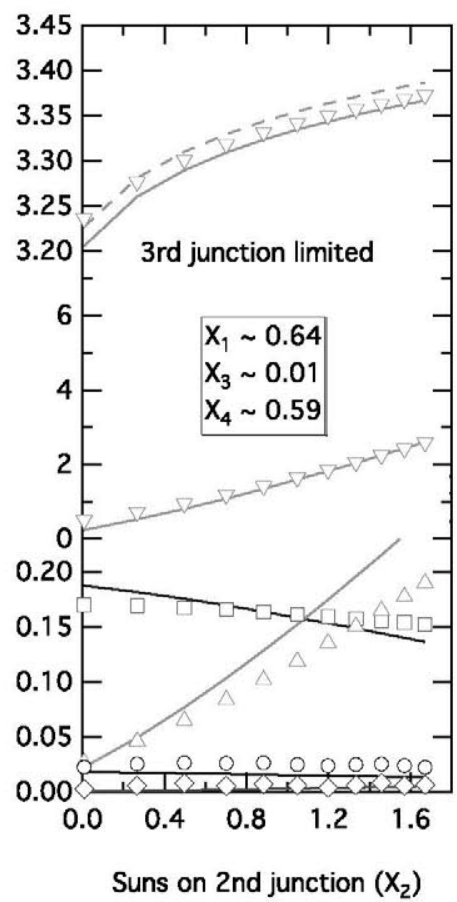

(b)

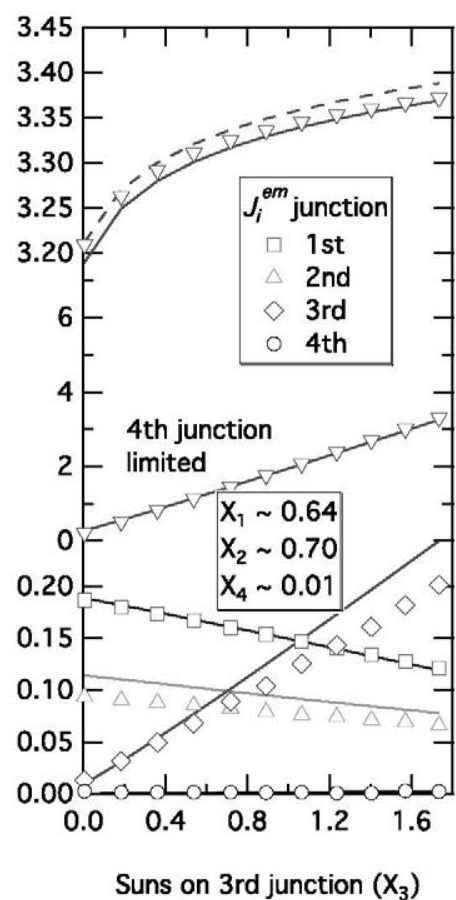

(c)

Fig. 6. "Dark" varied illumination measurements. Total $V_{\mathrm{oc}}$, the limiting photocurrent $=J(2.7 \mathrm{~V})$, and light emitted from each junction, $J_{i}^{e m}$, is shown as the external illumination is varied while intentionally limiting a particular junction by leaving that junction in the dark. The inset legends show the approximate illumination on the junctions that are not intentionally varied. Data (markers) fit using parameters in Table I (solid lines). The $V_{\text {oc }}$ is overestimated when the dark area correction is not performed (dashed lines). (a) First junction varied, second junction limited. (b) Second junction varied, third junction limited. (c) Third junction varied, fourth junction limited.

around approximately 0.7 suns on each junction. (The choice of 0.7 suns is rather arbitrary but is roughly half the maximum light obtainable using the LEDs in this system.) The limiting photocurrent in these graphs was measured as the current density at $2.7 \mathrm{~V}$ rather than $0 \mathrm{~V}$ in order to force the fourth junction to be limiting because $2.7 \mathrm{~V}$ is slightly above the onset of the $\mathrm{RBB}$ in the fourth junction (described in detail below). The slope of the limiting photocurrent as the illumination on that limiting junction is varied is very sensitive to $J_{i}^{1 X}$. (This region is shown in the left portion of each graph in Fig. 5.) We note that the external subcell photocurrents calculated from the integrated $\mathrm{EQE}, J_{i}^{\mathrm{QE}} \equiv \int \mathrm{EQE}_{i}^{\mathrm{DUT}}(\lambda) \phi^{\mathrm{AM} 1.5 \mathrm{~d}}(\lambda) d \lambda$ that are also listed in Table I, were used as initial guesses and are comparable to the model fit $J_{i}^{1 X}$ within $\sim 1 \mathrm{~mA} / \mathrm{cm}^{2}$ giving some idea of the uncertainties. The total series-connected $V_{\text {oc }}$ is also shown in Figs. 5 and 6 , but this was primarily fit using the EL data. Note that only when $J_{i}^{1 X}$ and $J$ are corrected by the ratio of illuminated to total area as discussed previously is the excellent agreement in the $V_{\text {oc }}$ achieved.

The LC behavior of each junction is particularly accentuated by illuminating each junction EXCEPT the limiting junction while varying the light to the junction above it, as shown in Fig. 6. While $J_{i}^{01}$ and $J_{i}^{0 n}$ are actually more sensitively determined from EL measurements (especially because EL can be more easily performed at higher and lower current densities), they contribute to the nonlinearity of the LC. The overall magnitude of the limiting photocurrent is sensitively fit in Fig. 6 by adjusting $\beta_{k i}$.
The presence of shunts and RBB in junctions complicates the fitting procedure significantly because in these cases, the $J_{\mathrm{sc}}$ of the multijunction solar cell is then not necessarily the limiting photocurrent. RBB is particularly common in low bandgap junctions, such as Ge and 0.7-eV InGaAs, but this complication can be overcome by measuring the current between the onset of breakdown and the maximum power point [11], [35] to estimate the limiting photocurrent of that "leaky" junction. The shape of the RBB and shunt can be observed (and fit) in the $J-V$ curves by significantly limiting that junction during illumination to fit the shunt conductance and RBB parameters. Several full $J-V$ curves are shown in Fig. 7 for the example 4J IMM where the fourth junction is the limiting photocurrent. A distinct RBB characteristic is observed, which is fit making use of (14). The actual photocurrent in the fourth-junction limited case can be measured by using the current density at a voltage above the onset of RBB ( $2.7 \mathrm{~V}$ in this case). Once the RBB is well characterized, the model accurately predicts $J(V)$ at every voltage. In this example, no significant voltage-independent shunting was observed, either in intentionally junction limited $J-V$ curves or the EL and dark $J-V$ curves, where they become obvious with a linear slope.

\section{Measurement of Emitted Light While Varying External Illumination on Each Junction Independently}

The mechanism of light emission (voltage-driven EL or voltage-independent PL) that gives rise to LC is not obvi- 


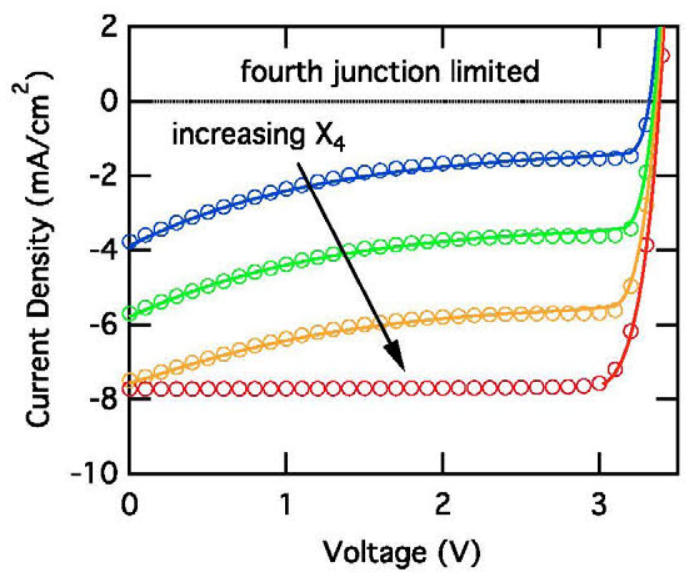

Fig. 7. Light $J-V$ curves with fourth junction limited showing RBB of the fourth junction until another junction becomes limiting (red). Data (markers) and fit (lines).

ous from the previously outlined measurements. Indeed, the voltage-independent PL contribution had not been recognized until recently [33]. When the $J_{\mathrm{sc}}$ of a multijunction solar cell is measured while a particular junction is limiting as described in the previous subsection, the limiting junction is in reverse bias $\left(V_{\lim }<0\right)$, while all other junctions are in forward bias $\left(V_{i} \approx V_{i}^{O C}\right)$. Thus, any LC that occurs and results in a change of the limiting junction photocurrent always includes both voltagedriven EL and voltage-independent PL components making it difficult to separate the effects. Any light emitted from the limiting junction (which is in reverse bias) comes only from the voltage-independent PL mechanism, but will never contribute LC to the measured $J_{\mathrm{sc}}$, because the junction to which it is transferred is, by construction, not the limiting junction. Therefore, a measurement of the light emitted from the limiting junction while varying only the external illumination on that limiting junction is the best way to quantify $\gamma_{i}$ in individual junctions of multijunction solar cells. This measurement can be taken simultaneously with the LC measurements from Section III-B as long as the wavelengths of the external light sources are chosen so that they do not overlap with the wavelengths of the emitted light. $J_{i}^{\mathrm{em}}$ is measured as outlined in Section III-A, while $X_{i}$ is varied and quantified as outlined in Section III-B. It is important during this measurement that any nearby devices that are left. at open-circuit are masked to prevent inadvertent detection of their higher PL than the intended device at lower voltages.

When the first junction of this $4 \mathrm{~J}$ IMM is limiting, as in Fig. 8(a), there is a significant increase in the emission from the first junction as the illumination on the first junction is increased, confirming the importance of the voltage-independent PL emission in this junction. The effect can also be seen in the left side of the bottom panel of Fig. 5(a), where the integration of the emitted spectra is also observed to increase even though the first junction is in reverse bias. (Some spectral overlap with the 740-nm LED light makes good absolute quantification difficult.) Once the first junction is no longer limiting in Fig. 5(a), the slope of that increase changes, indicating an additional contribution from the EL portion. Likewise, Fig. 8(b) corresponding to the left side of Fig. 5(b) shows a strong effect of
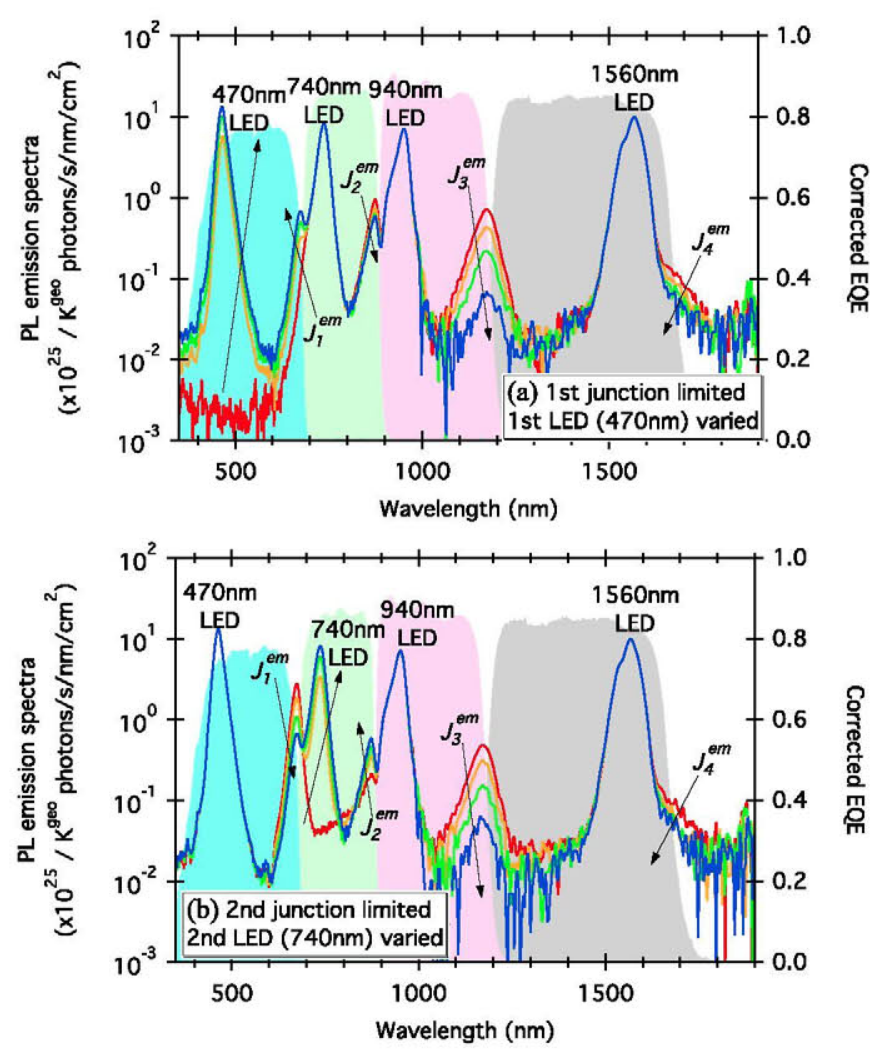

Fig. 8. Emission spectra (and reflection of LED illumination) for various LED illumination intensities while (a) the first junction is limiting and (b) the second junction is limiting. The colored lines indicate spectra under different LED illumination as one LED is varied. The arrows show increasing LED illumination and the resulting increasing or decreasing PL emission, as quantified in Fig. 5(a) and (b). The EQE is shown in the background for reference.

TABLE II

SHORT-CIRCUIT PL RATIO $\gamma_{i}$ FOR A VARIETY OF SINGLE-JUNCTION SOLAR CELLS

\begin{tabular}{lccccc}
\hline \hline Eg $(\mathrm{eV})$ & Material & Junction design & Growth direction & Absorber doping & $\gamma_{i}$ \\
\hline 1.8 & GaInP & RHJ & Inverted & n-type & 0.0062 \\
1.8 & GaInP & Traditional & Inverted & p-type & 0.0002 \\
1.4 & GaAs & RHJ & inverted & n-type & 0.0036 \\
1.4 & GaAs & Traditional & upright & p-type & 0.0001 \\
\hline \hline
\end{tabular}

the voltage-independent PL emission from the second junction. Fitting of the slope of $J_{i}^{\mathrm{em}}$ as $X_{i}$ is varied when junction $i$ is limiting is sensitive to the parameter $\gamma_{i}$ in multijunction characterization. Since this voltage-independent PL is a second-order effect relative to the much stronger voltage-driven EL, and spectral overlap can be difficult to remove entirely, $\gamma_{i}$ can be difficult to quantify accurately within multijunction devices using the simple integration over finite limits as described by (15). Improved quantification could be achieved by a more complex fitting of the various emission peaks.

Given these uncertainties, $\gamma_{i}$ is more easily observed as the slope of $J_{1}^{\mathrm{em}}$ versus $J_{\mathrm{sc}}$ in single-junction isotypes as described in [33]. Table II lists the results of several designs of single-junction devices for comparison with the results in Table I. The magnitude of $\gamma_{i}$ is also affected by its optical 


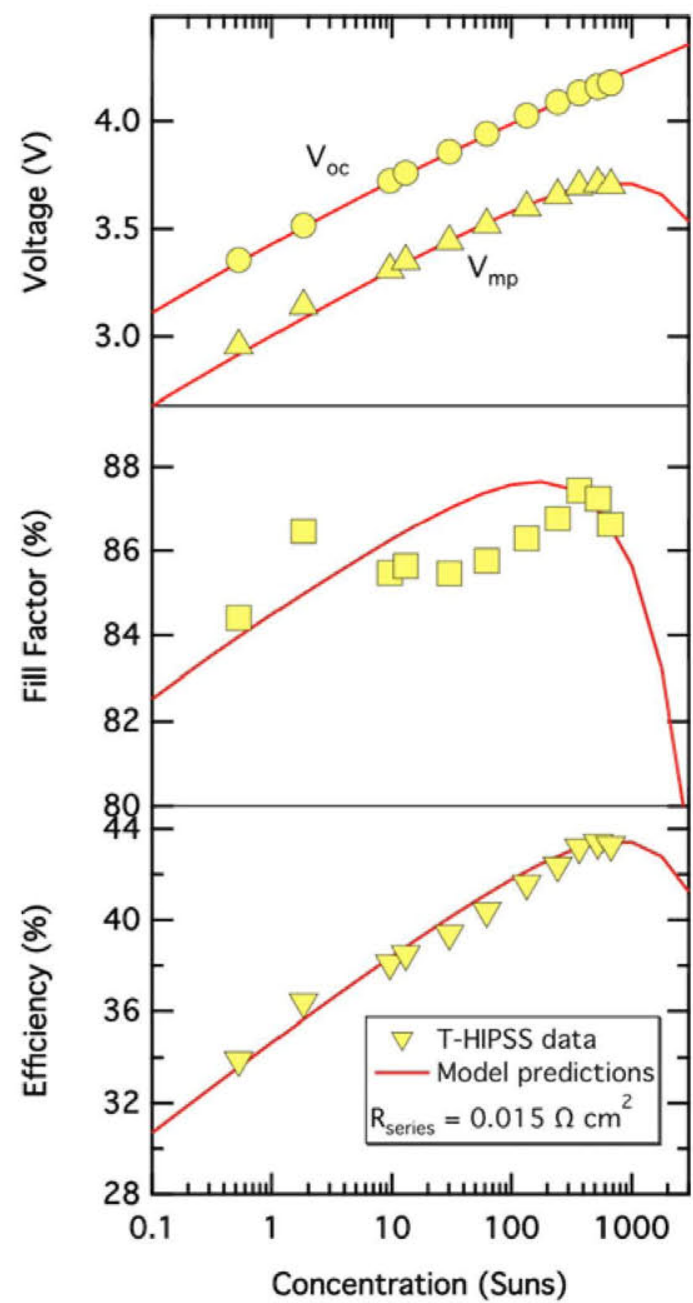

Fig. 9. T-HIPSS concentrator flash measurements (markers) and fit predictions (lines) of 4J IMM device characterized in previous sections. Only the series resistance was fit again here, as discussed in the text. The measured photocurrent ratios $\left\{X_{i} / X_{1}\right\}=\{1.0: 1.002: 1.001: 0.991\}$ were also used for the model fit. Measurements assume linearity of concentration and maximum power with the one-sun short-circuit current as determined from the model.

environment (e.g., BSR); therefore, the single-junction value cannot be taken directly from this table to use in multijunction fitting. However, in general, these values illustrate that a junction design with a predominately n-type absorber, such as a rear-heterojunction design [42], [48], has much higher $\gamma_{i}$ as predicted due to its lower minority carrier mobility [31]. While $\gamma_{i}$ may be difficult to detect in multijunction solar cells without measuring the emission directly, its effect on the modeled cell performance can be pronounced.

\section{Concentrator flash Measurements}

A good simultaneous fit of all the experimental data of the previous three subsections should completely characterize any series-connected multijunction solar cell, but the model's real usefulness lies in its ability to predict the performance under other conditions such as high-concentration flash measurements and varying real-world outdoor spectra. Comparing these predictions to flash measurements is only valid if the flash measure- ments are well characterized. Previous generations of concentrator flash simulators were not spectrally adjustable for more than two junctions [53], but a recently available tunable highintensity solar simulator (T-HIPSS) can be adjusted fairly well to $\left\{X_{1}, X_{2}, X_{3}, \ldots\right\}=\{C, C, C, \ldots\}$ over a wide range of concentrations, C. More importantly, the complete characterization of $\left\{X_{i}\right\}$ is at least attempted. In Fig. 9, we show a comparison of the predictions of this model to the T-HIPSS flash measurements of the device over a range of concentrations. The voltages and efficiency as a function of concentration predicted by this model using only the parameters determined previously (except $R^{\text {ser }}$ ) fit the flash data quite well. Unfortunately, the fill factor measured here as a function of concentration is quite noisy, obscuring the expected trends that the model shows. The approximate magnitude of the fill factor, however, is in good agreement with the predictions of the model.

When this model fits flash data well, it also provides insight into how the device is operating under these conditions and the sensitivity to the input parameters. For example, the model can indicate which junction is limiting under given input conditions and how much the fill factor may be changed when the nonlimiting junction is overilluminated. It is important to note again here that the lumped series resistance under highintensity illumination is not well predicted by the high current density forward-bias dark $J-V$ measurements due to the differing three dimensional current paths between electrically and optically generated current and the asymmetry of the heterojunction barrier resistances [54]. In these measurements, current was extracted from two busbars on opposite sides of the grid during flash measurements but only using one busbar during dark $J-V$ measurements, further exacerbating the difference in the fitting of the lumped series resistance here.

\section{CONCLUSION}

The complex behavior of series-connected multjunction solar cells has been described by a comprehensive optoelectronic model that incorporates two important mechanisms for light emission: series and shunt resistances and multiple realistic recombination mechanisms. An empirical fitting to this model using a finite number of electrical and optical laboratory characterization measurements is outlined and used to predict performance under arbitrary operating conditions. This model can facilitate improved understanding of high concentration measurements and will allow improved predictions of energy production in varying real-world environments.

\section{ACKNOWLEDGMENT}

The authors gratefully acknowledge $\mathrm{M}$. Green of the University of New South Wales for pointing out the likely importance of short-circuit PL. The 4J IMM was grown and processed by W. Olavarria, M. Young, A. Duda, and S. Ward.

\section{REFERENCES}

[1] R. M. France et al., "Quadruple junction inverted metamorphic concentrator devices," IEEE J.. Photovoltaics, vol. 5, no. 1, pp. 432-437, Jan. 2015. 
[2] R. M. France and J. F. Geisz. NREL demonstrates $45.7 \%$ efficiency for concentrator solar cells. (2014). [Online]. Available: http://www.nrel.gov/news/press/2014/15436.html

[3] F. Dimroth. New world record for solar cell efficiency at $46 \%$. (2014). [Online]. Available: http://www.ise.fraunhofer.de/en/press-andmedia/press-releases/press-releases-2014/new-world-record-for-solarcell-efficiency-at-46-percent

[4] F. Dimroth et al., "Wafer bonded four-junction $\mathrm{GaInP} / \mathrm{GaAs} /$ GaInAsP/GaInAs concentrator solar cells with $44.7 \%$ efficiency," Prog. Photovoltaic, Res. Appl., vol. 22, pp. 277-282, 2014.

[5] D. Ding, S. R. Johnson, W.-Q. Yu, and Y.-H. Zhang, "A semi-analytical model for semiconductor solar cells," J. Appl. Phys., vol. 110, p. 123104, 2011.

[6] U. Rau, "Reciprocity relation between photovoltaic quantum efficiency and electroluminescent emission of solar cells," Phys. Rev. B, vol. 76, no. 8, p. 085303 , Aug. 2007.

[7] O. D. Miller, E. Yablonovitch, and S. R. Kurtz, "Strong internal and external luminescence as solar cells approach the Shockley-Queisser limit," IEEE J. Photovoltaics, vol. 2, no. 3, pp. 303-311, Jul. 2012.

[8] C. Baur, M. Hermie, F. Dimroth, and A. W. Bett, "Effects of optical coupling in III-V multilayer systems," Appl. Phys. Lett., vol. 90, p. 192109. 2007.

[9] H. Yoon, R. R. King, G. Kinsey, S. Kurtz, and D. D. Krut, "Radiative coupling effects in GaInP/GaAs/Ge multijunction solar cells," in Proc. 3rd World Conf. PV Energy Convers., Osaka, Japan, 2003, pp. 745-748.

[10] D. J. Friedman, J. F. Geisz, and M. A. Steiner, "Effect of luminescent coupling on the optimal design of multijunction solar cells," IEEE $J$ Photovoltaics, vol. 4, no. 3, pp. 986-990, May 2014

[11] M. A. Steiner et al., "Measuring IV curves and subcell photocurrents in the presence of luminescent coupling," IEEE J. Photovoltaics, vol. 3 , no. 2 , pp. 879-887, Apr. 2013.

[12] D. J. Friedman, J. F. Geisz, and M. A. Steiner, "Analysis of multijunction solar cells current-voltage characteristics in the presence of luminescent coupling," IEEE J. Photovoltaics, vol. 3, no. 4, pp. 1429-1436, Oct. 2013.

[13] M. A. Steiner and J. F. Geisz, "Non-linear luminescent coupling in seriesconnected multijunction solar cells," Appl. Phys. Lett., vol. 100, no. 25 , p. 251106, Jun. 18, 2012.

[14] D. Derkacs, T. Bilir, and V. A. Sabnis, "Luminescent coupling in GaAs/GaInNAsSb multijunction solar cells," IEEE J. Photovoltaics, vol. 3, no. 1, pp. 520-527, Jan. 2013.

[15] K.-H. Lee et al., "Demonstration of photon coupling in dual multiplequantum-well solar cells," IEEE J. Photovoltaics, vol. 2, no. 1, pp. 68-74, Jan. 2012.

[16] S. H. Lim, J.-J. Li, E. H. Steenbergen, and Y.-H. Zhang, "Luminescence coupling effects on multijunction solar cell external quantum efficiency measurement," Prog. Photovoltaics, vol. 21, no. 3, pp. 344-350, 2011.

[17] J. Jia et al., "Bias-dependence of luminescent coupling efficiency in multijunction solar cells," Opt. Exp., vol. 23, no. 7, pp. A219-A231, 2015.

[18] S. C. Choo, "Carrier generation-recombination in the space-charge region of an asymmetrical p-n junction," Solid-State Electron., vol. 11, pp. 1069 1077,1968

[19] C. T. Sah, R. N. Noyce, and W. Shockley, "Carrier generation and recombination in p-n junctions and p-n junction characteristics," Proc. IRE, vol. 45, pp. $1228-1243,1957$.

[20] A. L. Fahrenbruch and R. H. Bube, Fundamentals of Solar Cells Photovoltaic Solar Energy Conversion. New York, NY, USA: Academic, 1983.

[21] M. A. Steiner et al., "Optical enhancement of the open-circuit voltage in high quality GaAs solar cells," J. Appl. Phys., vol. 113, no. 12, art. no. 123109, Mar. 28, 2013.

[22] B. M. Kayes et al., "27.6\% conversion efficiency, a new record for singlejunction solar cells under 1 sun illumination," in Proc. 37th IEEE Photovoltaic Spec. Conf., Seattle, WA,USA, 2011, pp. 4-8.

[23] I. Garcia et al., "Back reflectors based on buried $\mathrm{Al}_{2} \mathrm{O}_{3}$ for enhancement of photon recycling in monolithic, on-substrate III-V solar cells," Appl. Phys. Lett., vol. 105, art. no. 133507, 2014

[24] I. García et al., "Design of semiconductor-based back reflectors for high Voc monolithic multijunction solar cells," in Proc. 38th IEEE Photovoltaic Spec. Conf., 2012, pp. 4-8.

[25] M. P. Lumb, M. A. Steiner, J. F. Geisz, and R. J. Walters, "Incorporating photon recycling into the analytical drift-diffusion model of high efficiency solar cells," J. Appl. Phys., vol. 116, art. no. 194504, 2014.

[26] V. M. Andreev, V. A. Grilikhes, and V. D. Rumyantsev, Photovoltaic Conversion Concentrated Sunlight. Chichester, U.K.: Wiley, 1997.

[27] R. Hoheisel et al., "Electroluminescence analysis of irradiated GaInP/GaInAs/Ge space solar cells," Sol. Energy Mater. Sol. Cells, vol. 108, pp. 235-240, Jan. 2013 .
[28] S. Roensch, R. Hoheisel, F. Dimroth, and A. W. Bett, "Subcell I-V characteristic analysis of $\mathrm{GaInP} / \mathrm{GaInAs} / \mathrm{Ge}$ solar cells using electroluminescence measurements," Appl. Phys. Lett., vol. 98, no. 25, art. no. 251113, Jun. 20, 2011.

[29] T. Kirchartz et al., "Internal voltages in GaInP/GaInAs/Ge multijunction solar cells determined by electroluminescence measurements," Appl. Phys. Lett., vol. 92, no. 12, art. no. 123502, 2008.

[30] J. F. Geisz, R. M. France, I. Garcia, M. A. Steiner, and D. J. Friedman, "Device characterization for design optimization of 4 junction inverted metamorphic concentrator solar cells," in Proc. AIP Conf., art. no. 114, vol. 1616, 2014.

[31] D. Lan and M. A. Green, "Analytical expressions for spectral composition of band luminescence from silicon solar cells under optical and electrical bias," Appl. Phys. Lett., vol. 104, art. no. 173902, 2014.

[32] U. Rau, "Superposition and reciprocity in the electroluminescence and photoluminescence of solar cells," IEEE J. Photovoltaics, vol. 2, no. 2, pp. 169-172, Apr. 2012.

[33] D. Lan et al., "Improved modeling of photoluminescent and electroluminescent coupling in multijunction solar cells," Sol. Energy Mater. Sol. Cells, vol. 143, pp. 48-51, 2015.

[34] M. Meusel et al. "Spectral response measurements of monolithic GaInP/Ga(In)As/Ge triple-junction solar cells: Measurement artifacts and their explanation," Prog. Photovoltaics, Res. Appl., vol. 11, no. 8, pp. 499-514, 2003

[35] E. Barrigón, P. Espinet, Y. Contreras, and I. Rey-Stolle, "Implications of low breakdown voltage of component subcells on external quantum efficiency measurements of multijunction solar cells," Prog. Photovoltaics, Res. Appl., 2015.

[36] C. R. Osterwald, K. A. Emery, D. R. Myers, and R. E. Hart, "Primary reference cell calibrations at SERI: History and methods," in Proc. 21st IEEE Photovoltaic Spec. Conf., Kissimmee, FL,USA, 1990, pp. 1062 1067.

[37] A. Marti, J. L. Balenzategui, and R. F. Reyna, "Photon recycling and Shockley's diode equation," J. Appl. Phys., vol. 82, no. 8, pp. 4067-4075, 1997.

[38] E. D. Kosten, J. H. Atwater, J. Parsons, A. Polman, and H. A. Atwater, "Highly efficient GaAs solar cells by limiting light emission angle," Light: Sci. Appl, vol. 2, p. ed45, 2013.

[39] E. S. Toberer, A. C. Tamboli, M. Steiner, and S. Kurtz, "Analysis of solar cell quality using voltage metrics," in Proc. IEEE 38th Photovoltaic Spec. Conf., 2012, pp. 001327-001331.

[40] R. R. King et al., "Band gap-voltage offset and energy production in next-generation multijunction solar cells," Prog. Photovoltaics, Res. Appl., vol. 19 , no. 7, pp. 797-812, 2011.

[41] M. A. Steiner et al. "Effects of internal luminescence and internal optics on $V_{\circ C}$ and $J_{\mathrm{SC}}$ of III-V solar cells," IEEE J. Photovoltaics, vol. 3, no. 4, pp. 1437-1442, Oct. 2013.

[42] J. F. Geisz et al., "Implications of redesigned, high-radiative-efficiency GaInP Junctions on III-V multijunction concentrator solar cells," IEEE J. Photovoltaics, vol. 5, no. 1, pp. 418-424, Jan. 2015.

[43] C. H. Henry, "Limiting efficiencies of ideal single and multiple energy gap terrestrial solar cells," J. Appl. Phys., vol. 51, no. 8, pp. 4494-4500, 1980

[44] W. Shockley and H. J. Queisser, "Detailed balance limit of efficiency of p-n junction solar cells," J. Appl. Phys., vol. 32, p. 510, 1961.

[45] H. J. Hovel, "Carrier collection, spectral response, and photocurrent," in Semiconductors and semimetals, vol. 11, R. K. Willardson and A. C. Beer, Eds. Orlando, FL, USA: Academic, 1975, eqs. (15), (19), and (20).

[46] D. L. King, B. R. Hansen, J. M. Moore, and D. Aiken, "New methods of measuring performance of monolithic multijunction solar cells," in Proc. 28th IEEE Photovoltaic Spec. Conf., Anchorage, AK, USA, 2000, pp. 1197-1201.

[47] M. C. Alonso-García and J. M. Ruíz, "Analysis and modelling the reverse characteristic of photovoltaic cells," Sol. Energy Mater. Sol. Cells, vol. 90 , pp. 1105-1120, 2006.

[48] J. F. Geisz, M. A. Steiner, I. Garcia, S. R. Kurtz, and D. J. Friedman, "Enhanced external radiative efficiency for $20.8 \%$ efficient single-junction GaInP solar cells," Appl. Phys. Lett., vol. 103, art. no. 041118, 2013.

[49] G. Siefer, C. Baur, and A. W. Bett, "External quantum efficiency measurements of germanium bottom subcells: measurement artifacts and correction procedures," in Proc. IEEE Photovoltaic Spec. Conf., Honolulu, HI, USA, 2010, pp. 704-707.

[50] J.-J. Li, S. H. Lim, C. R. Allen, D. Ding, and Y.-H. Zhang, "Combined effects of shunt and luminescence coupling on external quantum efficiency measurements of multijunction solar cells," IEEE J. Photovoltaics, vol. 1, no. 2 , pp. 225-230, Oct. 2011 
[51] J.-J. Li, C. R. Allen, S. H. Lim, and Y.-H. Zhang, "Elimination of artifacts in external quantum efficiency measurements of multijunction solar cells using a pulsed voltage bias," IEEE J. Photovoltaics, vol. 3, no. 2, pp. 769-775, Apr. 2013.

[52] J.-J. Li and Y.-H. Zhang, "Elimination of artifacts in external quantum efficiency measurements for multijunction solar cells using a pulsed light bias," IEEE J. Photovoltaics, vol. 3, no. 1, pp. 364-369, Jan. 2013.

[53] C. R. Osterwald, M. W. Wanlass, T. Moriarty, M. A. Steiner, and K. A. Emery, "Empirical procedure to correct concentrator cell efficiency measurement errors caused by unfiltered Xenon flash solar simulators," in Proc. 40th IEEE Photovoltaic Spec. Conf., Denver, CO, USA, 2014, pp. 2616-2619.

[54] J. M. Olson, M. A. Steiner, and A. Kanevce, "Using measurements of fill factor at high irradiance to deduce heterobarrier band offsets," in Proc. 37th Photovoltaic Spec. Conf., Seattle, WA, USA, 2011, pp. 003754-003757.

Authors' photographs and biographies not available at the time of publication. 